CORRIGENDUM

\title{
Nuclear landscape of HIV-1 infection and integration
}

Marina Lusic and Robert F. Siliciano

Nature Reviews Microbiology 15, 69-82 (2017)

In the first paragraph of the section 'Sequence specificity and chromatin determinants', at the end of the sixth sentence an incorrect reference is cited. In the following sentence accurate information is now provided in regard to what was done in the original study. These sentences should read as follows: "A recent study of approximately 1 million integration sites in infected HEK 293 cells showed that $75 \%$ of integrations occurred in active, RNA pol II-dependent transcriptional units that had numerous introns; when corrected for their relative length, no selection of intronic over exonic sequences was observed $^{79}$. Analysis of transcriptional units that were grouped based on their number of introns revealed that integration density correlates strongly with the levels of splicing, and that the cellular protein LEDGF is required for targeting highly spliced transcriptional units, through its direct interaction with numerous splicing factors ${ }^{79}$." The authors apologize to the readers for any misunderstanding caused. 